\author{
Voix plurielles \\ Volume 5, Numéro 1 : mai 2008
}

\title{
Isabelle Dangy
}

\section{De la chambre au chapitre : l'hotel virtuel de Suite à l'hotel crystal}

Citation MLA : Dangy, Isabelle. «De la chambre au chapitre : l'hotel virtuel de Suite à l'hotel crystal.» Voix plurielles 5.1 (mai 2008).

(C) Voix plurielles, revue électronique de l'APFUCC 2008. 


\title{
De la chambre au chapitre : l'hotel virtuel de Suite à l'hotel crystal
}

\author{
Isabelle Dangy \\ Cierec De Saint-Étienne
}

Mai 2008

\section{Résumé}

Suite à l'hôtel Crystal (2004) d'Olivier Rolin propose quarante-trois cellules romanesques contiguës consacrées chacune à la description d'une chambre d'hôtel suivie du récit d'un épisode, généralement loufoque, survenu lors du séjour du narrateur dans cette chambre. Ce texte ironique invite à réfléchir sur les enjeux littéraires liés à l'identification de la chambre et du chapitre, sur l'effet provoqué par une écriture de la reprise et de la variation, et sur le sens de l'édifice virtuel construit par la juxtaposition en un même lieu romanesque de chambres situées en des points dispersés de la planète.

Il existe une analogie parlante entre une nuit passée dans une chambre d'hôtel et la lecture d'un livre. Ces deux institutions sont en effet vouées à l'accueil de visiteurs passagers, anonymes, successifs et qui ont, la plupart du temps, payé. A partir d'une série d'éléments partiellement prévisibles mais promis à de nombreuses variations (d'un côté le lit, le mobilier de base, la zone vouée aux ablutions, et de l'autre les pages, les paragraphes et les différents codes génériques qui assurent le fonctionnement du pacte de lecture), la chambre d'hôtel et le livre, le roman surtout, proposent à leurs usagers un compromis à chaque fois négociable entre la clôture du refuge et le rêve, le voyage, l'escapade ou, éventuellement, le commerce amoureux. La lecture ouvre une parenthèse spatiale et temporelle dans le quotidien du lecteur, de même que le séjour dans une auberge ou un palace. Mais que l'on pousse un peu plus loin la métaphore, et c'est l'hôtel lui-même, avec la variété de ses chambres, de ses couloirs, de ses espaces d'accueil et de ses sas de transition, qui entre en équivalence avec la littérature.

Lorsque Olivier Rolin écrit en 2004 Suite à l'hôtel Crystal, il exploite avec méthode la séduction de cette analogie, puisque le roman se compose, après un avertissement attribué à l' «Éditeur », de quarante-trois chapitres, ou, si l'on préfère, de quarante-trois «chambres » occupées tour à tour par la mémoire du narrateur et offertes à l'imagination du lecteur qui se voit entraîné dans cette circumnavigation. Le titre de chaque chapitre correspond à un numéro d'ordre suivi d'une localisation, par exemple, pour le chapitre 12, "Chambre 402, Dan Chu Hôtel, 29 Phô Trang Tien, Hanoi », ou encore, pour le chapitre 24, "Chambre 417, Cecil Hôtel, Saad Zaghloul square, Alexandrie », tandis que la table des matières se confond avec la récapitulation 
méthodique de ces adresses. Cependant l'édifice qui abrite toutes ces chambres, contiguës entre elles dans le volume du livre, est un établissement imaginaire, car les différentes adresses réfèrent à des lieux dispersés aux quatre coins de la planète, de Coimbra à Bucarest, de Port-Saïd à Krasnoïarsk, de Montélimar à Vancouver.

Certes, l'ensemble fonctionne comme une construction autobiographique loufoque et déconstruite, si l'on en croit différentes indications semées dans le texte, qui poussent avec une complaisance ironique en direction de cette hypothèse. On serait en quelque sorte devant un Grand Hôtel de la mémoire pourvu d'au moins une chambre en forme de trou. Le titre même, qui joue sur la polysémie du mot «suite », lequel peut désigner une série (de chapitres) aussi bien qu'un appartement luxueux dans un hôtel, est une invitation à comprendre l'œuvre dans ce sens. En outre, le dernier chapitre est significativement consacré à l' "Hôtel du Point final » (Rolin 228) appellation inattendue qui contraste avec d'autres désignations plus conventionnelles telles que Hôtel Opera, Hôtel Astor, Hôtel Hilton Bonaventure.

Mais pour le lecteur qui pénètre chapitre après chapitre dans les chambres, quel est le sens de chacun de ces brefs séjours ainsi que du parcours accompli ? L'opération qu'on attend de lui consiste-t-elle à reconstruire une histoire en reliant tant bien que mal les épisodes, en faisant la part du vraisemblable, du fantasme et du canular, autrement dit en défaisant le désordre savant du roman ? Ou au contraire doit-il plutôt se loger au hasard, nuit après nuit, dans cet hôtel qui est en même temps ici et là, et où les repères temporels sont en général éludés ?

\section{Précédents occupants}

Les remarques qui vont suivre se donnent pour fin de débrouiller quelques-uns des enjeux littéraires liés à l'assimilation du chapitre à la chambre et du livre à l'hôtel. Mais, puisque en ce genre de lieux les pièces sont occupées tour à tour par de nombreux clients, autant chercher d'abord les traces laissées par eux sur la coiffeuse ou dans les plis des draps... traces intertextuelles, bien entendu : Suite à l'hôtel Crystal est habité par de nombreuses références littéraires explicites qui contribuent à définir le projet de l'auteur. Leur quantité interdit de les recenser ici, mais leur mode d'insertion peut être brièvement évoqué. Le roman s'appuie sur le prétexte traditionnel du manuscrit trouvé dans une valise égarée : ce manuscrit est en fait constitué de fragments écrits par leur auteur sur divers supports qui vont du revers d'un menu de restaurant aux marges d'un billet de concert, en passant par des feuilles arrachées aux œuvres d'écrivains célèbres, comme c'est le cas dans le chapitre 1, pourvu de l'indication finale «Texte manuscrit sur trois pages de garde arrachées des Misérables, de Victor Hugo, éd. Laffont, coll. «Bouquins » (19).

Le procédé fournit à Olivier Rolin l'occasion de rendre hommage à différents auteurs, tels que Volodine, Cendrars, Poe, Tchekhov, Whitman, etc. Les allusions revêtent souvent un caractère ludique : le chapitre 12, consacré à une macabre histoire d'orang-outang, est griffonné sur les pages prétendument arrachées à une édition des Histoires extraordinaires, en référence évidemment à la nouvelle «Double assassinat dans la rue Morgue » d'Edgar Poe. Nul doute que d'autres allusions plus ténues, y compris des allusions intratextuelles à d'autres œuvres d'Olivier Rolin, se dissimulent au fil du texte, mais le procédé des pages de garde, associé à d'autres éléments du paratexte, met en valeur la référence la plus éclatante de Suite à l'hôtel Crystal, à savoir l'œuvre de Georges Perec. Ainsi le manuscrit du chapitre 21 aurait été écrit sur « les pages 
blanches laissées à la demande de l'auteur, à la suite de Je me souviens, de Georges Perec, Hachette, Librairie du XX $X^{\mathrm{e}}$ siècle » (113). D'autres mentions plus longues interviennent, par exemple au cours du chapitre 20 où le mystérieux rédacteur des feuillets épars annonce l'intention de compléter et de mener à terme une œuvre inachevée du même auteur, œuvre dont le projet est évoqué sous le titre "fragments d'un travail en cours " à l'intérieur du chapitre intitulé «la chambre » dans Espèces d'espaces (Perec 31-6). Il s'agissait pour Perec de rédiger une sorte d'autobiographie nocturne en établissant une liste descriptive des «lieux où [il a] dormi ». La forme de Suite à l'hôtel Crystal reprendrait donc, en l'amplifiant et en le modifiant, ce programme perecquien dont la tendance à assimiler l'espace clos de la chambre à celui de l'écriture est déjà évident.

Mais en réalité une autre source perecquienne trahit sa présence dans la forme donnée au livre : il s'agit bien entendu de La Vie mode d'emploi, « romans » dont les quatre-vingt-dix neuf chapitres cernent la vie présente et passée d'un immeuble parisien imaginaire, et dont l'une des lectures possibles, lecture immobilière en quelque sorte, consiste en une exploration guidée des différents étages, escaliers, caves et corridors. L'importance assignée à cette source intertextuelle annonce l'intention de se situer dans le sillage d'au moins un écrivain tenté par les exercices descriptifs et narratifs qu'induit l'assimilation du livre et de l'immeuble. Il n'est pas indifférent que cet écrivain ait été intéressé aussi par la dialectique de l'éparpillement et de la réunion, et travaillé par le désir de loger sous un toit unique ces errants que sont souvent ses personnages. L'empreinte profonde du travail perecquien apparaît donc dans Suite à l'hôtel Crystal, mais associée à un traitement particulier et restrictif qui consiste dans le remplacement de l'immeuble par l'hôtel.

L'analyse de cet espace peut être menée d'abord à l'échelle de la chambre, saisie isolément. On s'intéressera ensuite à l'effet de variation qu'engendre la succession des quarante-trois pièces. On interrogera enfin le sens de l'édifice imaginaire constitué en fin de parcours.

\section{La chambre, un lieu de rendez-vous}

Chacune des chambres où le lecteur pénètre à la suite du narrateur forme une cellule bien circonscrite. En effet, un protocole respecté avec une grande régularité préside à la composition de chaque chapitre : l'aspect des lieux est décrit avec un luxe de détails dont la précision touche à la technicité, puis une deuxième partie, de longueur variable, est consacrée au récit d'un épisode, souvent loufoque, que le narrateur a vécu dans la chambre ou à proximité immédiate de celle-ci. On peut reprendre l'exemple du chapitre 12, dont quatre pages environ décrivent une chambre à Hanoi tandis que les deux dernières pages racontent comment le narrateur, au sortir de cette chambre, s'y est pris pour kidnapper, au musée d'histoire naturelle de cette même ville, le squelette d'un orang-outang qui ne serait en fait que la dépouille maquillée d'un officier américain abattu pendant la guerre du Viêt-Nam.

La plupart du temps, la minutie avec laquelle sont évoqués les murs, la disposition des meubles et des objets, les proportions de la pièce, ne cherche à suggérer aucun rapport logique avec la nature des événements dont elle est le cadre : description et narration fonctionnent chacune de son côté, en deux temps bien distincts, et même si aucun intervalle typographique ne matérialise ces deux moments de la séquence, un hiatus les sépare du point de vue du contenu. La description proprement dite, menée au présent de l'indicatif, commence fréquemment par 
quelques données numériques précisant les dimensions de la pièce, la hauteur de plafond, puis la couleur des murs et des sols. Ensuite, le regard du scripteur parcourt méthodiquement la chambre en suivant les murs et en citant les objets qui y sont appuyés ou accrochés, jusqu'à revenir à son point de départ. S'il y a lieu, une station dans l'entrée, la salle de bain, ou sur le balcon peut agrémenter ce tour d'horizon. Les couleurs et les matières sont dépeintes avec insistance. Ce procédé systématique a tout d'abord un caractère englobant : les contours de la chambre sont nettement posés, comme pour stabiliser un espace distinct de ce qui l'entoure, et s'il arrive que des objets ou bibelots soient soumis à un examen attentif, ce sont cependant les murs qui fournissent la base descriptive. Il en résulte un effet de cloisonnement qui se répercute sur le projet romanesque en refermant chaque chapitre sur lui-même. Il s'agit à chaque fois de construire un espace protégé, où la mention des plans, des choses, des proportions permet de créer la réalité par sa nomination, de conjurer peut-être un sentiment d'inconsistance, comme si tous les éléments du décor désignaient, en convergeant vers elle, une conscience ou du moins un état de conscience qui se confond ainsi avec les lieux jumeaux du chapitre et de la chambre.

La description semble d'autre part à la recherche d'une objectivité maximale. Les objets sont énumérés d'un point de vue fonctionnel, sans qu'aucun d'eux soit gratifié d'une importance particulière. Olivier Rolin pousse jusqu'à l'extrême la neutralité et le désir d'exhaustivité explorés par certains romanciers des années soixante (on peut penser à certaines pages de L'Inquisitoire de Pinget), comme pour en repousser les limites. La description semble également conçue pour illustrer certaines analyses théoriques développées dans $\mathrm{Du}$ descriptif par Philippe Hamon, qui voit sous toute description le schéma d'une nomenclature destinée à développer un hyperonyme, en l'occurrence celui de la chambre (Hamon 127-63).

En réalité il s'agit pourtant ici d'une fausse neutralité, car il arrive fréquemment que les termes employés contiennent un jugement esthétique direct : « de chaque côté de la tête de lit, au dessus de quoi est encadrée une chose affreuse représentant un vase de lys blancs, une table de chevet porte une lampe à pétrole munie d'une ampoule électrique, et, pour celle de droite, un téléphone à touches ivoire de marque iwatsu » (41). Ou incluent des appréciations à peine voilées : «Une chaise à haut dossier et un fauteuil à tendance ellipsoïde (enfin...), tendus d'un tissu rose gerbeux traversé de lignes pointillées blanches, complètent le mobilier» (26). Chaque description devient de la sorte le lieu d'une rencontre avec le lecteur, rencontre faite de connivence et de perplexité car, si les évaluations humoristiques font sourire, le procédé descriptif ne livre pas ses clés. Où le descripteur veut-il en venir ? Quel est le sens de cette accumulation de détails peu signifiants, qui produit un effet quasi hypnotique dont on observe avec intérêt l'installation? Plus les notations sont précises et nombreuses, moins il est possible de visualiser vraiment l'espace de la chambre, et plus la lecture provoque une réaction anesthésique qui n'est pas de l'ennui mais un indéfinissable plaisir textuel fait d'une adhésion gratuite et prolongée aux phrases, aux mots.

Ce phénomène se complique encore dans la seconde partie du chapitre, lorsque la description cède la place à un bref récit dont l'origine se situe dans la mémoire du narrateur. Ce qui, à la première lecture, apparaissait comme une description neutre, objective et gratuite, se transforme alors en souvenir: la chambre est remémorée à cause de l'histoire à laquelle elle est métonymiquement associée, même si elle ne lui sert pas de décor immédiat. Le regard jeté précédemment autour de la pièce paraissait celui que l'on pose sur un lieu inconnu dont on énumère les composantes au fur et à mesure qu'on les découvre; de cette observation distanciée 
on passe désormais à l'intimité relative de la mémoire et du récit, si bien que le sens de la description s'obscurcit encore rétrospectivement. Le fait que les épisodes relatés baignent dans l'invraisemblance et la parodie, tout en contenant certainement des allusions cryptées à la vie de l'auteur, redouble la perplexité (agréable) du lecteur. Le chapitre, comme la chambre, enferme l'intime et l'anonyme, le fonctionnel et la fabulation dans une même enceinte où se confrontent, le temps d'une visite, l'auteur de l'écriture et l'auteur de la lecture. La chambre est ainsi le lieu privilégié d'une écriture de la connivence.

\section{Quarante-trois variations hôtelières}

Un tel rituel ne se produit pas une fois mais plus de quarante. Le même lecteur est convié à une série de rendez-vous successifs qui infléchissent peu à peu ses réactions, car assez rapidement se crée un effet d'attente que le texte se plait à satisfaire et à divertir au moyen de plaisantes variations. L'identification du chapitre à la chambre entraîne une écriture itérative qui se situe du côté de l'incantatoire et de la poésie, ou, mieux encore, du conte. Ainsi la majorité des chapitres commence, logiquement et métaphoriquement, par une formule consacrée à la porte : «La porte d'entrée, en bois sombre, ouvre dans un couloir d'environ 1,5x3m » (106), « La porte à double battant, en bois enduit d'une peinture ancienne, écaillée, entre le gris plomb et vert bronze, munie d'un grand loquet de fer coulissant horizontalement et d'une petite poignée de cuivre ronde, se trouve au fond d'une antichambre » (82), etc. La porte est à la fois l'embrayeur descriptif, le point de départ du tour d'horizon spatial évoqué plus haut, le symbole de l'ouverture sur un monde parallèle et clos, et l'objet répété qui manifeste une écriture de la série, de la variation, de la saturation.

Cependant il faut noter qu'une double visée sociologique intervient ici, même si elle ne constitue pas l'enjeu primordial de l'œuvre. Tout d'abord il s'agit de réduire à un nombre fini d'éléments l'ensemble qui correspond à une chambre d'hôtel: le lit, les tables de nuit, les lampes, l'installation sanitaire, le réfrigérateur, la télévision... Cet ensemble reflète le voyageur moderne, dont les besoins minimaux et les dépendances inconscientes sont dessinés en creux par le décor qui l'accueille. Il amène à réfléchir sur le concept même de chambre d'hôtel comme raccourci d'une civilisation mondialisée. D'autre part, selon le pays où se voit située la chambre, les modalités de détail diffèrent, et Olivier Rolin se plait à insister sur l'ameublement exagérément cossu d'un hôtel suisse, sur l'allure délabrée et vieillotte d'une chambre sibérienne, ou plus généralement sur la niaiserie et le conformisme de certains aménagements décoratifs.

Parmi les exercices de variation auxquels se livre l'auteur, l'ordre de présentation joue un rôle important. Tantôt (c'est le cas le plus général), la description précède le récit qui, comme on l'a vu, fonctionne à part. Tantôt l'écrivain entame un récit in medias res qui s'interrompt pour laisser place à un paragraphe descriptif et reprend ensuite, tantôt il supprime la partie descriptive, jouant avec le modèle auquel il a habitué le lecteur. A ce jeu contribuent aussi quelques chambres « aberrantes », étrangères à ce modèle, et sur lesquelles on reviendra plus loin.

Parmi les objets dont la récurrence cimente au contraire autour du Même le tournoiement de l'Autre, figurent deux éléments particuliers : la fenêtre et le miroir, qui offrent deux issues dans l'enceinte de chaque chambre. A de rares exceptions près, la description inclut en effet le paysage que l'on aperçoit entre les montants d'une baie vitrée : une échappée sur le monde extérieur. Cela suffit-il pour atténuer l'effet d'enfermement créé par l'inventaire des murs et des 
objets ? A peine, car, même si une certaine variété caractérise ces paysages (un tarmac enneigé en Sibérie, le lac Léman, les caténaires du tramway à Coimbra, un parking à Miami Beach, l'étagement des toits de Buenos Aires, etc.) et même si l'on sent de temps à autre se réveiller le lyrisme réprimé de l'auteur, ils sont la plupart du temps traités sur le même mode énumératif et dépourvu d'affect que l'aménagement intérieur des chambres, au point de se confondre avec celui-ci, comme s'il s'agissait de tableaux accrochés au mur plutôt que de brèches pratiquées dans le continuum de la description maniaque. A Düsseldorf, par exemple, un même paragraphe juxtapose les éléments suivants, qui peuvent donner une idée de la méthode adoptée par Olivier Rolin :

La partie centrale du mur du fond est percée par les deux panneaux de la fenêtre, que sépare un montant assez large: chacun, à huisserie de métal laqué blanc et doubles vitrages, mesure environ $2 \mathrm{~m}$ de haut sur $70 \mathrm{~cm}$ de large. A travers, on aperçoit, au-delà d'un espace bas et obscur (un petit parking, puis, plus loin, probablement des cours) un rang de petits immeubles et de maisons dont les toits se découpent, noirs sur le ciel bleu sombre. Fenêtres allumées. Un haut clocher noir surplombe ça, avec à droite un building illuminé, couronné de feux rouges, et une cheminée d'usine, et à gauche le sommet de la tour de la télé, au bord du Rhin, minaret techno nimbé de lumière bleue et clignotant de feux rouges. Des rideaux de voilage blanc coulissent sur toute la longueur du mur, ainsi que des doubles rideaux de toile plastifiée saumon pâle. A gauche de la fenêtre, un radiateur en tôle laquée blanche. (212)

D'autre part, il est fréquent qu'un miroir rompe la monotonie du discours descriptif : celui-ci renvoie alors l'image du descripteur qui s'y reflète, image généralement dépeinte en termes ironiques assez peu flatteurs, et qui oscille entre la suggestion d'une intériorité et l'étrangeté caractéristique de ces autoportraits où l'on se regarde comme si l'on était un autre. En quelques occasions, il arrive que le regard du personnage explore les deux issues d'un seul coup et perçoive dans le miroir, derrière ses propres traits, la perspective du paysage livré par la fenêtre. Cet alignement des deux ouvertures est évidemment une des possibilités de la combinatoire mise en place. Quoi qu'il en soit, une tonalité obsessionnelle tempérée d'ironie résulte de ce mode descriptif fondé sur un regard tournant qui balaie comme un phare la réalité insignifiante de la chambre.

A un deuxième niveau, qui n'intervient qu'au bout de quelques chapitres, la chambre, prêt-àporter d'une intimité mensongère, négation du voyage dans le voyage même, se propose donc comme image de la littérature, faux lieu d'ouverture dont les apparentes issues sont bloquées et ne donnent que sur le moi ou l'illusoire, où la réalité et sa représentation se confondent par principe, où l'invention doit chaque jour reconquérir son territoire menacé par le ressassement des stéréotypes. Cette image pourrait sembler sombre, malgré le ton jovial, n'était la présence d'un phénomène qui dépasse le caractère sériel de l'écriture et donne, par le biais d'un montage raffiné, une dimension polyphonique à Suite à l'hôtel Crystal. On peut en prendre conscience en 
deux temps, dont le premier tient à l'existence, de plus en plus fréquente au fur et à mesure que l'on progresse vers la fin du volume, d'exceptions à la règle établie au départ.

\section{Les chambres aberrantes}

Les quelques chapitres qui échappent au schéma général prennent par contraste un relief particulier. Ce sont en premier lieu ceux qui tournent autour de l'hôtel Crystal, rue de Chanzy, à Nancy, et qui se distinguent des autres d'abord par un retour sur le même lieu, ensuite par un refus de décrire. Ainsi le chapitre 13 commence, à la place habituelle du temps descriptif, par le mot phrase «Rien» (71), tandis que le chapitre 17 se présente comme un fragment d'interrogatoire où le narrateur nie tout souvenir de cette chambre, et que, en deux autres chapitres, il a recours à une feinte, la première fois en masquant la chambre de l'hôtel Crystal sous l'apparence d'une autre, située à Arcachon, la deuxième fois en décrivant une chambre qu'il dénonce après coup comme fictive. Chambre du secret ou chambre de la mémoire défaillante, cette pièce vide (à l'exception de deux détails qui reviennent eux aussi de manière obsessionnelle comme des rescapés de l'oubli, une boîte de macarons et un porte-valises) forme le noyau autour duquel gravitent les autres chambres surchargées de notations, de meubles et d'objets. Par cet artifice de composition, Olivier Rolin mime ainsi, dans un esprit semi parodique, les dispositifs romanesques qui sont agencés autour d'un non-dit métaphorisé par une « chambre noire », une oubliette ou une salle interdite. Vue sous cet angle, la fusion de l'hôtel et du livre permet de matérialiser l'idée selon laquelle le désir de saturation et le vertige de la vacance se répondent comme les deux termes d'une littérature de la perte.

En second lieu, plusieurs autres hôtels se signalent par une enseigne insolite : hôtel Labyrinthe, hôtel Bellevues (dans ce cas c'est le pluriel qui alerte l'attention), hôtel du Départ, correspondant respectivement à la «chambre des portes », à la «chambre des fenêtres », à la « chambre des bruits ». A cette liste s'ajoute évidemment l'Hôtel du point final déjà mentionné. Les trois premiers établissements sont dépourvus d'adresse précise, et, au cas où le lecteur étourdi ne s'en serait pas fait la remarque, une note infra paginale explique à chaque fois que cette adresse est «illisible ». Leur absence de localisation et le thème qui les définit les logent à chaque fois dans le monde littéraire de la fable, cependant que la description se fait plus lyrique, comme si elle préparait le décollage du dernier chapitre, compendium poétique de chambres multiples, tourbillon d'éclats mémoriels semés avec exaltation au moment de clore le volume...

La chambre de l'hôtel Labyrinthe offre un panorama changeant de vestibules et de portes dont la dernière, après un épisode érotique, donne accès à un miroir. Celle de l'hôtel Bellevues est une improbable coupole percée de fenêtres qui donnent sur des paysages du monde entier, proposant en cela une image spéculaire du roman. La chambre des bruits, selon un procédé différent, introduit directement dans le champ d'une conscience qui s'éveille à l'univers sonore ambiant : en l'absence de toute description visuelle, le texte orchestre la réduction progressive du désordre sonore jusqu'à l'évocation d'un bruit unique et obsessionnel, celui d'une porte qui bat et répète indéfiniment l'écho d'un départ. Comme la série de l'hôtel Chanzy, ces chambres désignent des états de conscience, des espaces où s'effectue la jonction entre le monde extérieur et la réalité interne, l'arbitrage de la mémoire et de l'oubli. En outre la thématique qui leur est associée se répartit entre la mise en scène d'une profusion, d'un éclatement (les portes, les fenêtres, les bruits, la litanie du dernier chapitre) et la reprise d'une obsession qui surgit de 
dessous le multiple spectacle des choses et des paysages. Cette obsession est le fil qui relie entre eux les chambres et les chapitres, le fil autour duquel se construit en s'enroulant l'identité du narrateur. Celle-ci pourrait être le terme du récit, fût-ce sur le mode parodique.

\section{Dédoublement identitaire contrôlé}

L'identité du narrateur est soustraite à la curiosité du lecteur au début du livre, grâce à la fiction de l'Éditeur qui affirme publier des fragments manuscrits retrouvés dans les affaires d'un inconnu. La responsabilité de l'œuvre se divise ainsi entre le narrateur des fragments et les interventions de l'Éditeur, à savoir l'avertissement, l'index et l'appareil des notes, fréquentes et parfois développées, insérées au bas des fragments dont elles constituent un commentaire pseudo universitaire. Sous prétexte de souligner les bizarreries et de restaurer un minimum de logique ou d'esprit critique, ces interventions accroissent ironiquement le désordre et diluent l'identité du protagoniste.

Nonobstant ce procédé, la silhouette de celui-ci se dégage peu à peu. Les épisodes narratifs dessinent progressivement les contours d'une personnalité burlesque d'aventurier trafiquant chargé de missions extravagantes, un peu sur le retour, et enclin à se raconter avec une ironie bouffonne ; le retour régulier de certains personnages, par exemple ses alliés ou ses adversaires (les deux catégories pouvant aisément s'inverser) en matière de trafic international, ou encore les femmes de sa vie, contribue à unifier les fragments désordonnés de la mosaïque en donnant une relative cohérence à la personnalité du narrateur. Cohérence littéraire cependant, parce que construite à partir de stéréotypes et de traits parodiques. Mais parallèlement une deuxième image s'ébauche par le biais des descriptions étudiées plus haut: elle montre un personnage d'errant, une sorte de sans domicile fixe de la littérature dont l'entreprise serait de construire un paradoxe, un livre qui soit à la fois un reflet de son existence dispersée et un point d'ancrage imaginaire. Elle crée surtout, par accumulation, l'idée d'un personnage constamment exilé hors de sa sphère privée, condamné à scruter des décors perpétuellement étrangers - car qui s'acharnerait à décrire d'une manière aussi circonstanciée, et aussi distanciée, les couleurs et les objets de sa propre demeure ? De la somme des pièces envisagées se dégage l'impossibilité de l'intime, y compris lorsque le récit dévie vers des épisodes érotiques trop voisins du cliché pour évoquer une véritable expérience amoureuse, par exemple autour du thème de la femme de chambre troussée.

Enfin différentes allusions semées au fil des chapitres, de plus en plus fréquentes et explicites vers la fin, suggèrent la proximité de ce narrateur avec l'auteur lui-même. De cela il ne faudrait pas conclure que Suite à l'hôtel Crystal est une autobiographie tragi-comique. Deux considérations l'interdisent: d'une part Olivier Rolin ne prétend nullement avoir fait œuvre autobiographique ni même autofictionnelle mais semble plutôt s'amuser de ces subtilités génériques, et cela quand bien même il lui arrive de glisser des éléments vécus dans le parcours de son narrateur ou de faire référence à sa propre œuvre d'écrivain. D'autre part le ton n'est pas celui de la déploration ni de la gravité, mais, on l'a vu, celui de l'humour et de la connivence avec le lecteur. L'habitant des chambres successives ne nous fait pas visiter la maison de sa mémoire ni les différentes cases de son moi, mais ouvre une série de portes qui donnent sur autant de trompe-l'œil. 


\section{La tentation du simultanéisme}

Si ce n'est le moi, peut-être est-ce le monde que le livre souhaite évoquer. L'édifice virtuel qu'il bâtit pourrait être baptisé «Hôtel de la Planète ». Par la juxtaposition d'adresses, d'ambiances, de panoramas empruntés aux différents continents, Olivier Rolin cherche à donner forme à un rêve ancien de totalisation, qui consisterait à faire entrer la terre entière dans le roman. En cela il se situe dans une tradition proche du simultanéisme, dont Marie-Hélène Boblet et Dominique Viart ont étudié les prolongements dans la littérature contemporaine. Après avoir établi qu'au début du $\mathrm{XX}^{\mathrm{e}}$ siècle «la conscience d'un monde dont les lointains se sont trouvés soudain rapprochés par la facilité des transports et des communications. . marque les écrivains et les artistes bien au-delà des seuls groupes d'avant-garde »(Boblet et Viart 20), ils notent également que « la pratique simultanéiste caractérise ainsi la plupart des entreprises actuelles qui tiennent souvent du collage et de l'accumulation déhiérarchisée d'éléments hétérogènes offerts dans une même œuvre » (Boblet et Viart 43). Le simultanéisme ne se confond donc pas avec le récit ou la superposition d'événements concomitants, ni avec le refus du personnage central. Pris au sens large, ce terme s'applique à un certain nombre d'entreprises littéraires de notre époque visant soit à créer un réseau de sens entre divers lieux éclatés, soit à saisir la fragmentation dans une structure qui lui prête unité sans la figer. A ces deux titres, il concerne tout particulièrement Olivier Rolin, auquel font du reste allusion Marie-Hélène Boblet et Dominique Viart, en se référant notamment à L'Invention du monde, roman de 1993 assez proche de Suite à l'hôtel Crystal, où l'inspiration simultanéiste s'inscrit cependant dans l'enveloppe temporelle plus précise et plus ramassée d'une journée unique.

Une telle inspiration, dans la mesure où elle met une pluralité d'instants en équivalence, invite aussi à reconsidérer certains choix de lecture. Le procédé de succession des chambres contribue au même résultat. Certes il est toujours possible d'effectuer un parcours linéaire de la chambre 1 à la chambre 43, mais pourquoi ne pas traiter le livre comme un dictionnaire ou du moins un florilège, l'ouvrir au hasard et lire dans le désordre ? Les chemins à arpenter pour visiter une maison ne sont pas forcément programmés, donc, si un roman est bâti sur le modèle de l'immeuble, à plus forte raison sur celui de l'hôtel, l'attention du lecteur peut fort bien y musarder à son gré sans s'astreindre à telle ou telle progression. Certes, on l'a vu, un certain nombre d'arguments invite à respecter l'ordre selon lequel apparaissent les chambres, surtout vers la fin. Mais, dès l'avertissement, l'Éditeur informe le lecteur que cet ordre n'est pas garanti, qu'il relève d'un choix subjectif de sa part :

On n'a trouvé aucune indication explicite permettant d'établir un ordre parmi ces textes épars. Celui qui est proposé ici est donc entièrement de notre fait. . . En tout état de cause, plusieurs autres classements (peut-être même une infinité) seraient possibles. (11)

On peut sans doute entendre ces phrases comme une allusion à la mode littéraire des récits combinatoires ou interactifs, et un jeu avec celle-ci. Il n'en reste pas moins que l'interface livre/hôtel réactualise le débat sur la dialectique de la liberté et de la contrainte au cours de l'acte de lecture, sujet déjà mis en débat, d'une autre manière, par l'emploi d'une technique descriptive austère et réglée. 
L'intérêt de la littérature pour les chambres d'hôtel n'est pas nouveau. L'exercice descriptif qu'elles suscitent amusait déjà Chateaubriand voyageant à Waldmünchen en 1833. Point n'est besoin de s'appesantir sur la vertu dépaysante et l'étrangeté douloureuse, incompatible dans un premier temps avec l'intimité du sommeil, qu'elles acquièrent pour le narrateur de $A$ la recherche du temps perdu. Plus près de nous, la suggestion de personnages déracinés, inquiets, solitaires, passe dans les romans de Modiano par leur domiciliation instable dans de petits hôtels ou parfois dans des palaces. Les exemples pourraient être multipliés. Ils montreraient sans doute que la fréquence de la chambre d'hôtel dans le roman contemporain, en France et ailleurs, tient très simplement à sa modernité.

Peut-être appartient-elle, bien qu'il ne l'y inclue pas explicitement, à cette catégorie de lieux, caractéristique de l'espace contemporain, que Michel Foucault appelle contre emplacements ou hétérotopies, parce qu'ils sont «absolument autres que tous les emplacements qu'ils reflètent » (Foucault 756). Parmi les traits qu'il attribue à ces hétérotopies figurent en effet «le pouvoir de juxtaposer en un seul lieu réel plusieurs espaces, plusieurs emplacements qui sont en eux-mêmes incompatibles » (Foucault 758) et l'existence d'un «système d'ouverture et de fermeture qui, à la fois les isole et les rend pénétrables » (Foucault 760). Les chambres de Suite à l'hôtel Crystal possèdent clairement ces deux traits, mais il est aisé de constater que ceux-ci contribueraient tout autant à définir le livre, hétérotopie par excellence (bien que Foucault ne le mentionne pas non plus). Une telle modernité, définissant les lieux par le réseau de relations qu'ils entretiennent entre eux, recoupe les analyses de Michel Butor qui écrivait en 1964, pour situer les objectifs que devait selon lui poursuivre une écriture contemporaine :

Aujourd'hui, nous ne vivons jamais dans un lieu unique; nous avons toujours une localisation compliquée, c'est-à-dire que, lorsque nous sommes quelque part, nous pensons toujours aussi à ce qui se passe dans un autre endroit, nous avons des renseignements sur l'extérieur. Ouvrons la radio, nous voici en «présence» d'un speaker distant de centaines ou de milliers de mètres. Je suis bien «chez moi », mais ce «chez moi » n'est pas fermé, il communique par la radio, le téléphone, la presse, les livres, les œuvres d'art.

Saisir tout cela, donner tout cela, agir sciemment dans cet espace, le modifier par cet objet qu'est un livre parmi les autres meubles, meuble par excellence, «mobile» parmi les meubles. (Butor 71)

Ainsi, suivant ce conseil, l'écriture d'Olivier Rolin modifie, après en avoir fixé les contours, le robuste espace euclidien de la chambre en y incluant des lignes de fuite et en y suscitant en filigrane l'espace flou, implicite, indicible, qui auréole les personnages du récit. Il le transforme encore en le démultipliant, en lui adjoignant une temporalité qui louvoie entre le successif et le simultané. Enfin, parce que cette exploration topo-littéraire ne pouvait s'arrêter là et parce qu'il fallait une suite à Suite à l'hôtel Crystal, Olivier Rolin visite d'une autre manière encore le double espace de l'hôtel et du livre en proposant avec Rooms, en 2006, un recueil polyphonique où vingt-huit écrivains amis ou proches prolongent son entreprise en brodant à leur tour sur le 
thème de la chambre d'hôtel, et rendent de la sorte ce type d'établissement à sa vocation collective.

\section{BIBLIOGRAPHIE}

Boblet, Marie-Hélène et Viart, Dominique, «Esthétiques de la simultanéité », Jules Romains et les écritures de la simultanéité, Lille, Presses Universitaires du Septentrion, 1996.

Butor, Michel, «Philosophie de l'ameublement», Essais sur le roman, Paris, Gallimard (tel), 1964.

Foucault, Michel, «Des espaces autres », Dits et écrits, vol. 3, Paris, Gallimard (Quarto), 1984, $752-762$.

Hamon, Philippe, Du descriptif, Paris, Hachette (Supérieur), 1993.

Georges Perec, Espèces d'espaces, Paris, Galilée, 1974.

Olivier Rolin, Suite à l'hôtel Crystal, Paris, Seuil (La Librairie du XX ${ }^{\mathrm{e}}$ siècle), 2004. 